\title{
Case Report \\ Comprehensive Management of Severe Dental Fluorosis with Adhesively Bonded All-Ceramic Restorations
}

\author{
Jason D. Lee *, Natalie Inoue, Christine Lee, Sohyun Park and Sang J. Lee (iD \\ Department of Restorative Dentistry and Biomaterials Sciences, Harvard School of Dental Medicine, \\ Harvard University, Boston, MA 02115, USA; Natalie_inoue@hsdm.harvard.edu (N.I.); \\ Christine_lee@hsdm.harvard.edu (C.L.); Sohyun_park@hsdm.harvard.edu (S.P.); \\ Sang_Lee@hsdm.harvard.edu (S.J.L.) \\ * Correspondence: Jason_Lee@hsdm.harvard.edu
}

check for updates

Citation: Lee, J.D.; Inoue, N.; Lee, C.; Park, S.; Lee, S.J. Comprehensive

Management of Severe Dental Fluorosis with Adhesively Bonded All-Ceramic Restorations. Prosthesis 2021, 3, 194-208. https://doi.org/ $10.3390 /$ prosthesis 3030020

Academic Editor: Bruno Chrcanovic

Received: 21 May 2021

Accepted: 22 July 2021

Published: 26 July 2021

Publisher's Note: MDPI stays neutral with regard to jurisdictional claims in published maps and institutional affiliations.

Copyright: (c) 2021 by the authors. Licensee MDPI, Basel, Switzerland. This article is an open access article distributed under the terms and conditions of the Creative Commons Attribution (CC BY) license (https:/ / creativecommons.org/licenses/by/ $4.0 /)$.

\begin{abstract}
Dental fluorosis is a common disorder caused by excessive fluoride intake during tooth development. The esthetic consequences of dental fluorosis can negatively affect oral health-related quality of life and have lasting psychosocial effects. In severe cases, where the fluorosed enamel is prone to chipping, flaking, and developing caries, minimally invasive procedures are ineffectual and a more substantial restorative approach is required to restore optimal function and esthetics. However, no definitive guidelines exist for the management and treatment of severe dental fluorosis due to the limited evidence available in the literature. This case report describes the full-mouth rehabilitation of a patient with severe dental fluorosis utilizing adhesively bonded all-ceramic crowns, veneers, and overlays. The successful follow-up on this case indicates that adhesively bonded restorations may provide a viable option in the functional and esthetic management of severely fluorosed dentition.
\end{abstract}

Keywords: dental fluorosis; all-ceramic crowns; adhesive dentistry; full-mouth rehabilitation

\section{Introduction}

Dental fluorosis is caused by systemic overexposure to fluoride during tooth development [1]. The effects of excessive fluoride ingestion on developing teeth are related to time, dose, and duration of exposure, with several contributory mechanisms. These include fluoride-induced changes in the composition of the developing enamel matrix, as well as metabolic alterations to ameloblasts resulting in over-retention of secretory proteins [2]. In milder cases of dental fluorosis, the enamel is fully functional with the presence of opaque striations and a mottled appearance, while more severe cases are characterized by enamel surface porosity, pitting, and dark discoloration [3]. This porous enamel is more prone to chipping and flaking, with studies indicating a higher incidence of caries in individuals with severe dental fluorosis [4,5]. The structure of the underlying dentin can also be affected in severe cases, displaying a hypomineralized structure histologically [6] as well as a reduction in microhardness [7].

A variety of indices have been described in the literature that classify the severity of dental fluorosis. Thylstrup and Fejerskov, in 1978, proposed a classification that is commonly used today [3]. The Thylstrup and Fejerskov index (TFI) classifies the severity of dental fluorosis based on the clinical appearance of enamel (Table 1). This classification is considered to be biologically valid as it closely correlates the clinical features of the affected enamel to the histological features [8].

Local and systemic fluoride delivery through optimally fluoridated water supplies and fluoridated dentifrices has been associated with a decrease in caries' prevalence $[9,10]$. This caries-preventative effect can be attributed to the increased resistance to demineralization of fluorapatite as compared to hydroxyapatite. As described by Dean, maximum caries reduction is seen when $0.9-1.0 \mathrm{ppm}$ of fluoride is present in water supplies [11]. However, the increased usage of fluoride has also been associated with an increased prevalence of 
dental fluorosis $[9,10,12]$. Even at optimal levels of fluoridation, an estimated $48 \%$ of the population will present with some degree of dental fluorosis, with $12.5 \%$ manifesting into esthetic concerns [13]. However, a recent publication by Curtis and colleagues following a cohort of participants as part of the Iowa Fluoride Study concluded that both the prevalence and severity of mild to moderate fluorosis, as detected clinically, tend to decline during adolescence into young adulthood [14].

Table 1. Thylstrup and Fejerskov index (Fejerskov et al., 1988): mild (1-3), moderate (4-5), and severe (6-9).

\begin{tabular}{|c|c|}
\hline TFI & Criteria \\
\hline 0 & Normal translucency of enamel remains after prolonged air-drying. \\
\hline 1 & Narrow white lines located corresponding to perikymata. \\
\hline 2 & $\begin{array}{l}\text { Smooth surface: Pronounced lines of opacity that follow perikymata. Occasional confluence of adjacent lines. } \\
\text { Occlusal surface: scattered areas of opacity }<2 \mathrm{~mm} \text { in diameter and pronounced opacity of cuspal ridges. }\end{array}$ \\
\hline 3 & $\begin{array}{c}\text { Smooth surface: Merging and irregular cloudy areas of opacity. Accentuated drawing of perikymata often } \\
\text { visible between opacities. } \\
\text { Occlusal surface: Confluent areas of marked opacity. Worn areas appear almost normal but usually } \\
\text { circumscribed by a rim of opaque enamel. }\end{array}$ \\
\hline 4 & $\begin{array}{l}\text { Smooth surface: Entire surface exhibits marked opacity or appears chalky white. Parts of surface exposed to } \\
\text { attrition appear less affected. } \\
\text { Occlusal surface: Entire surface exhibits marked opacity. Attrition is often pronounced shortly after eruption. }\end{array}$ \\
\hline 5 & Entire surface displays marked opacity with focal loss of outermost enamel (pits) $<2 \mathrm{~mm}$ in diameter. \\
\hline 6 & $\begin{array}{l}\text { Smooth surface: pits are regularly arranged in horizontal bands }<2 \mathrm{~mm} \text { in vertical extension. } \\
\text { Occlusal surface: Confluent areas }<3 \mathrm{~mm} \text { in diameter exhibit loss of enamel. Marked attrition. }\end{array}$ \\
\hline 7 & $\begin{array}{l}\text { Smooth surface: loss of outermost enamel in irregular areas involving }<1 / 2 \text { of entire surface. } \\
\text { Occlusal surface: changes in the morphology caused by merging pits and marked attrition. }\end{array}$ \\
\hline 8 & Loss of outermost enamel involving $>1 / 2$ of surface. \\
\hline 9 & $\begin{array}{l}\text { Loss of main part of enamel with change in anatomic appearance of surface. Cervical rim of almost unafffected } \\
\text { enamel is often noted. }\end{array}$ \\
\hline
\end{tabular}

Endemic fluorosis remains a serious public health problem in developing countries of Africa and Asia, especially in regions corresponding with natural fluoride belts [15]. The ground water fluoride concentration is especially high in countries within the East African Rift Valley, including Kenya and Ethiopia. Fluoride concentrations of up to 2800 $\mathrm{mg} / \mathrm{l}$ have been reported in both surface and ground water in the Kenyan Rift Valley [16]. Fluoride exposure in these communities occurs not only from consumption of fluoride-rich groundwater, but also from various foods grown in soils with high fluoride content [17]. These factors, coupled with limited access to dental care, have led to lifelong dental health impacts in affected communities [18].

The esthetic consequences of dental fluorosis can negatively affect oral health-related quality of life and have lasting psychosocial effects on affected individuals [9]. Clinical presentation of moderate-severe fluorosis has been shown to be perceived as esthetically unpleasing in both adolescent and adult populations [19,20]. According to several studies evaluating the esthetic consequences of dental fluorosis, teeth with TFI scores of 3 or greater are considered to be of esthetic concern [19-21]. Treatment of these esthetic concerns is dependent on the extent of the fluorosis. Recommendations for the management of mild to moderate fluorosis include micro-abrasion, resin infiltration and bleaching techniques, either alone or in different combinations [22-24]. A recent systematic review by Di Giovanni et al. suggests resin infiltration as the most effective modality for esthetic improvement in cases of mild to moderate severity [24]. In severe cases where a large portion of the enamel structure has been compromised and caries is present, these minimally invasive procedures are ineffectual, and a more substantial restorative approach is required to restore optimal function and esthetics [25].

Due to the histomorphometric alterations that occur to enamel and dentinal structures with dental fluorosis [6,7], concerns have been raised about the bonding ability of resins to fluorosed enamel and dentin. In vitro studies suggest that, while shear bond strengths are lower with self-etch bonding systems, the impact of fluorotic enamel is much less 
when total-etch systems are utilized [26]. It has also been demonstrated that preparation of the enamel improves the bond strength between resin and enamel in fluorosed teeth [27]. On the other hand, an in vitro study by Ermis et al. determined that the bond strength achieved with a self-etch bonding system to moderately fluorosed dentin is similar to that achieved with normal dentin [28]. Thus, the researchers concluded that the degree of fluorosis has no significant effect on the bond strength achieved to dentin.

Although the treatment of mild to moderate fluorosis has been described and studied to a limited extent [22-24], guidelines for the management and treatment of severe dental fluorosis are greatly lacking in the literature. A case report by Farid et al. discussed the treatment of severe dental fluorosis with metal ceramic restorations in a full-mouth rehabilitation [29], but no publications to date have addressed the comprehensive treatment of this condition with bonded all-ceramic restorations. The use of bonded all-ceramic restorations allows for more conservative preparations, excellent esthetics, and proven longevity [30,31].

This case report presents the full-mouth rehabilitation of a patient with severe dental fluorosis utilizing adhesively bonded all-ceramic restorations with full coverage crowns, veneers, and overlays.

\section{Clinical Report}

A 24-year-old Kenyan male presented to the advanced graduate prosthodontics clinic at the XXX. He had grown up in Kiambu County in the former Central province of Kenya, which is an area known to contain elevated levels of fluoride in the ground water [16]. He was aware of the fluorotic condition of his teeth and expressed a desire to improve the esthetics of his smile. At full smile he displayed poor anterior esthetics due to fluorotic discoloration, staining, chipping, and asymmetry (Figure 1a,b). He also expressed concern with the long-term prognosis of his dentition due to the progressive discoloration and continued chipping of the enamel structure. The patient's medical history was unremarkable with no contraindications to dental treatment.

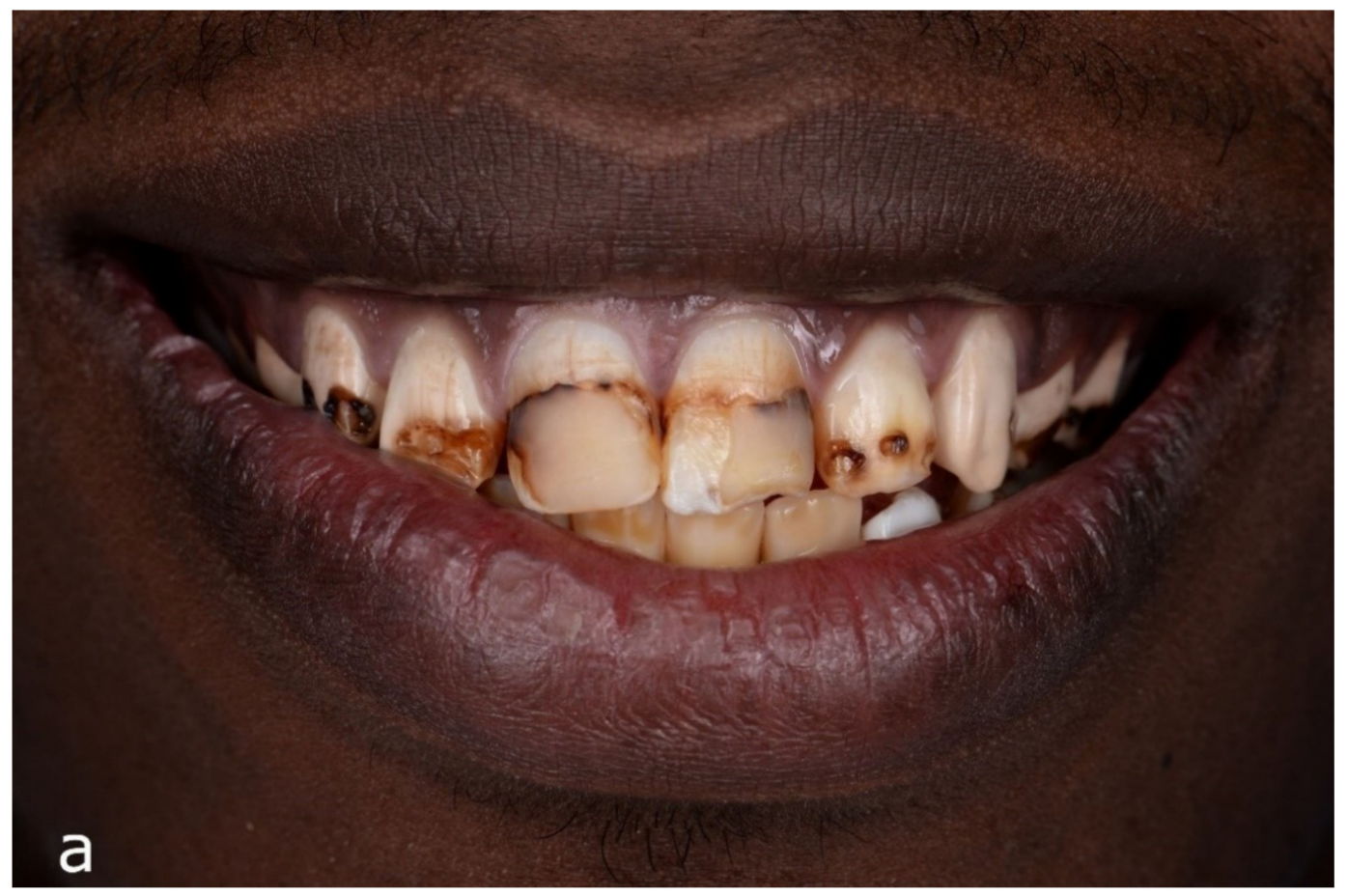

Figure 1. Cont. 


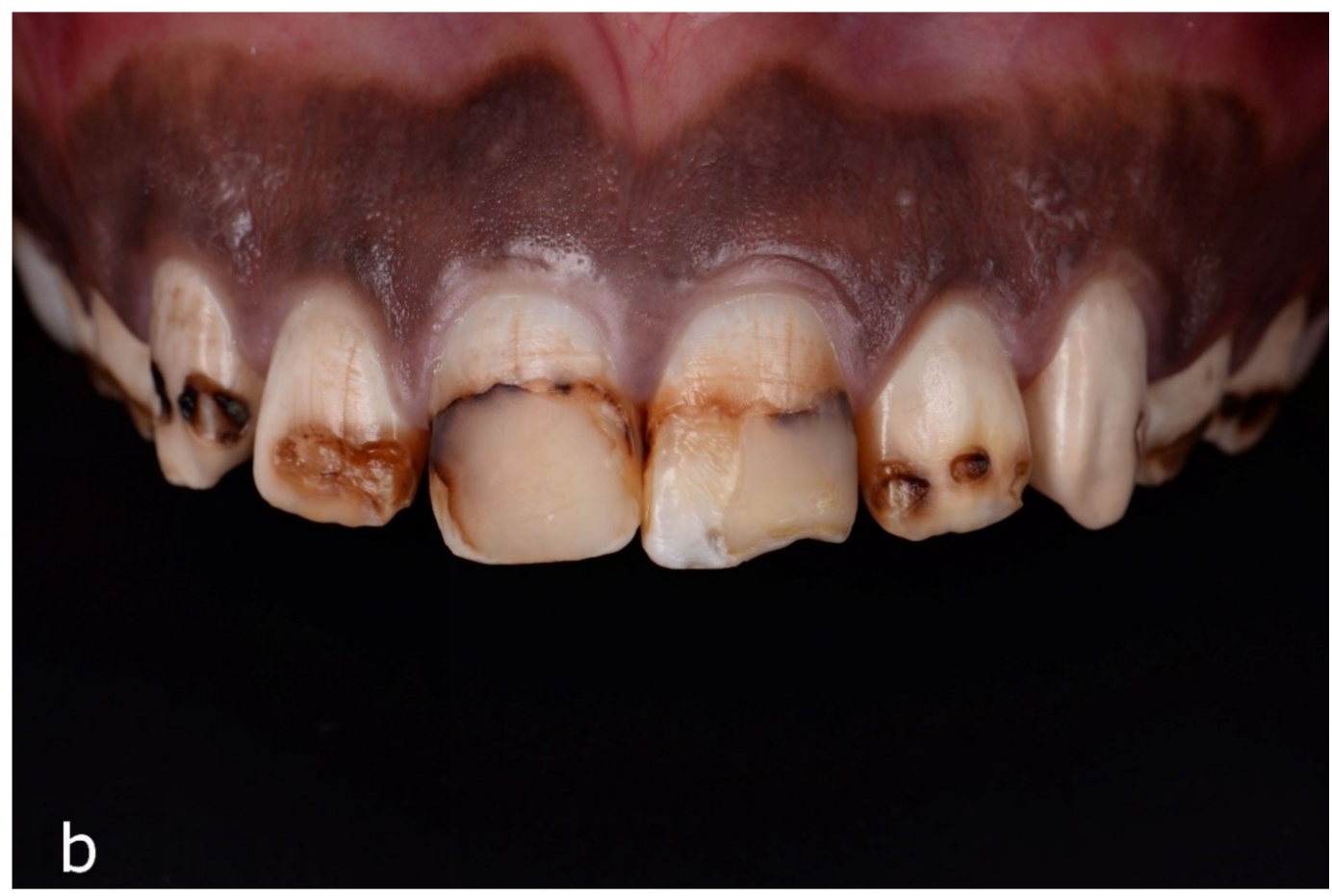

Figure 1. Extraoral view of pretreatment; (a) full smile and (b) retracted view of maxillary anterior dentition.

\subsection{Intraoral Examination}

The oral hygiene of the patient was excellent with minimal evidence of plaque accumulation. The gingiva was stippled with a darker coloration apically consistent with racial pigmentation. There were no periodontal probing depths greater than $3 \mathrm{~mm}$, and no bleeding on probing was recorded. The only restorations present were two failing composite restorations on the facial surfaces of the maxillary central incisors. Severe enamel fluorosis (TFI 6-7) was noted on a majority of the maxillary dentition characterized by irregular loss of enamel on the facial surfaces as well as attrition of the occlusal enamel on the posterior teeth. Several of the mandibular posterior teeth exhibited a similar presentation. The remaining teeth, with the exception of the third molars, exhibited enamel surface pitting consistent with a diagnosis of moderate fluorosis (TFI 5). Retracted intraoral views showed varying degrees of enamel fluorosis and failing composite restorations (Figure 2a-c). The mandibular incisor teeth displayed marked supra-eruption. Gingival margin discrepancies were noted between the maxillary anterior sextant and the posterior teeth due to altered passive eruption. A more extensive effect of fluorosis was noted on the maxillary arch (Figure 3a,b). However, all posterior teeth, with the exception of the third molars, displayed some degree of attrition with enamel pitting.

Angle Class I malocclusion was noted consisting of mild crowding along with supraeruption of the mandibular anterior teeth. The mandibular left first premolar was lingually positioned and in a scissor bite relationship with the opposing tooth. Altered passive eruption of the maxillary premolars was also observed. Infra-occlusion was noted on several posterior teeth due to enamel attrition, resulting in limited posterior occlusal stops. A full-mouth series of radiographs were exposed and revealed a healthy periodontal and endodontic status (Figure 4). Interproximal carious lesions were noted on multiple posterior teeth. 

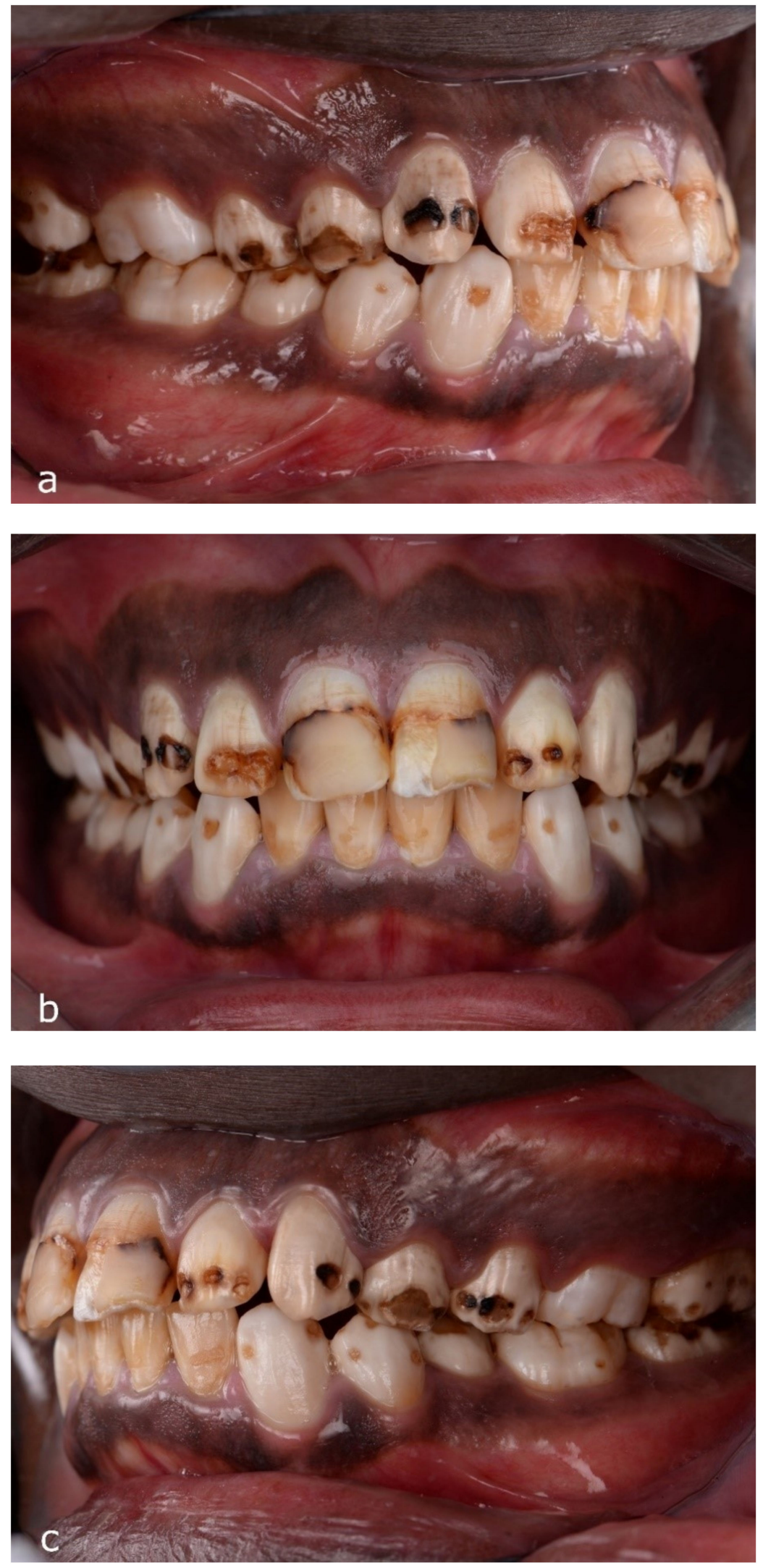

Figure 2. Retracted intraoral views of pretreatment: (a) right lateral view, (b) frontal view, and (c) left lateral view. 

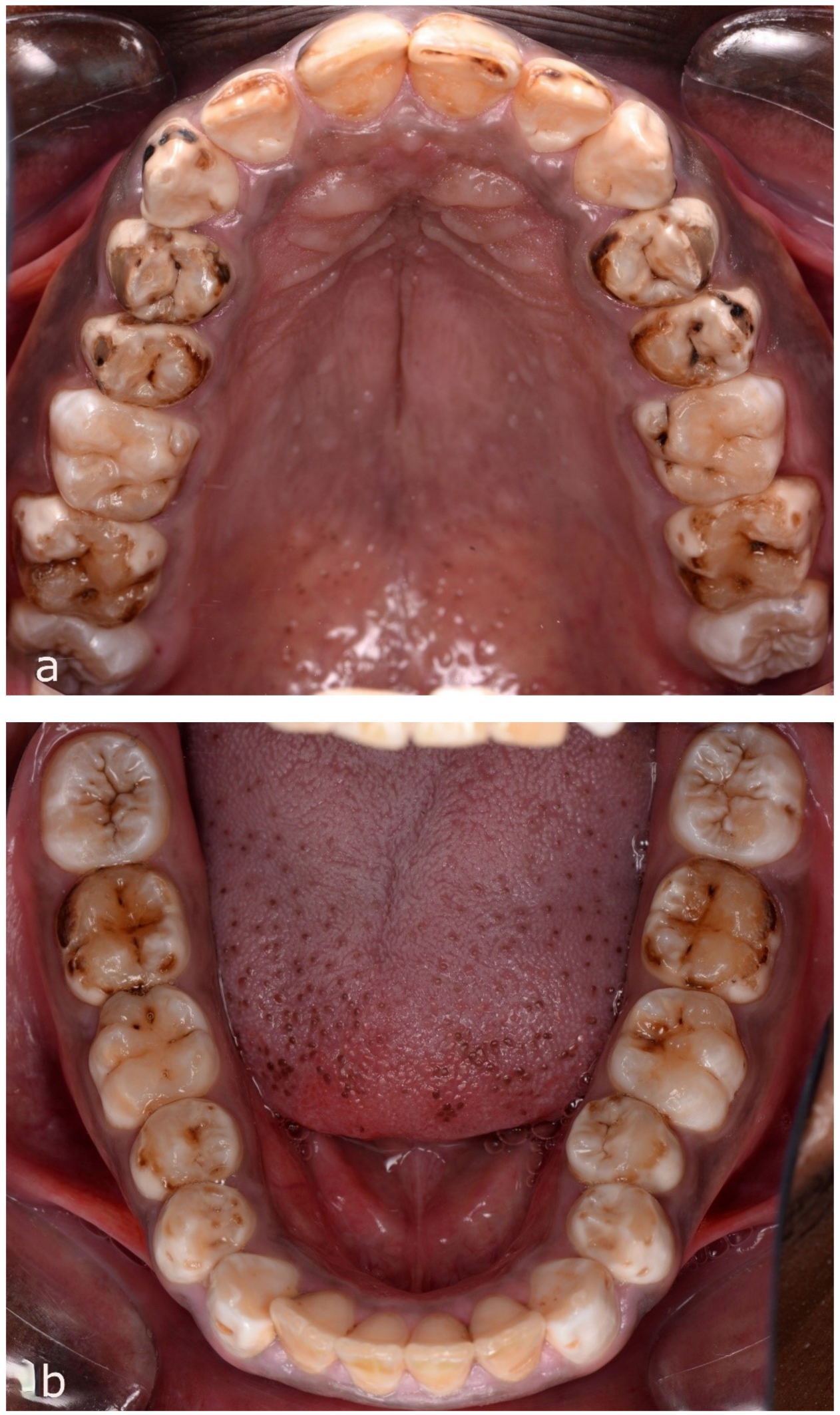

Figure 3. Occlusal views of pretreatment: (a) maxillary occlusal view and (b) mandibular occlusal view. 


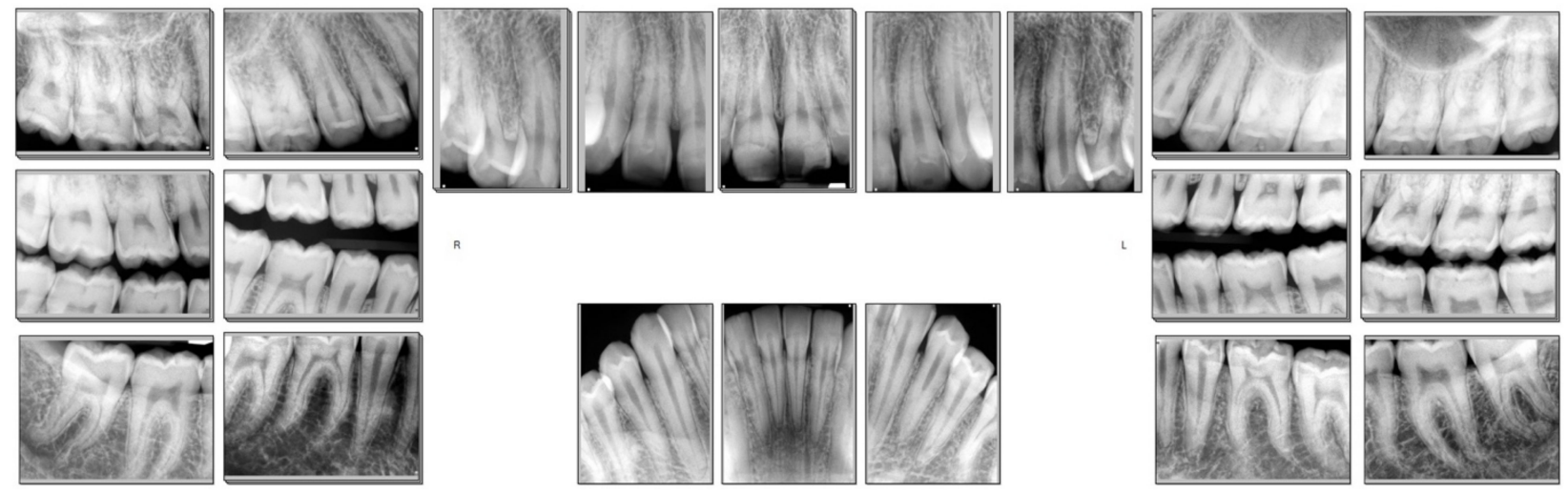

Figure 4. Pretreatment full-mouth X-rays. Interproximal caries is visible on multiple posterior teeth.

In consideration of the mild crowding and supra-eruption present, pre-prosthetic orthodontic treatment was recommended to the patient to idealize the occlusal plane and minimize tooth structure reduction. However, the patient declined this treatment due to the additional time and costs involved.

\subsection{Diagnostic Work Up}

Diagnostic impressions were taken and study models were mounted on a semiadjustable Artex CR articulator with a facebow record. A full-mouth wax up was then completed to idealize the contours of the future restorations (Figure 5). The existing vertical dimension of occlusion was maintained as an increase in the VDO was deemed unnecessary for successful treatment of the case, and the third molars were not to be restored. The supraerupted mandibular incisors were shortened to improve the occlusal plane and esthetics. The incisal edge of the left maxillary central incisor was used as an initial reference point for the maxillary wax up. The incisal edges of the maxillary centrals were lengthened about $0.5 \mathrm{~mm}$ in order to gain an adequate vertical overlap with the shortened mandibular anterior teeth as well as improve the overall esthetics.

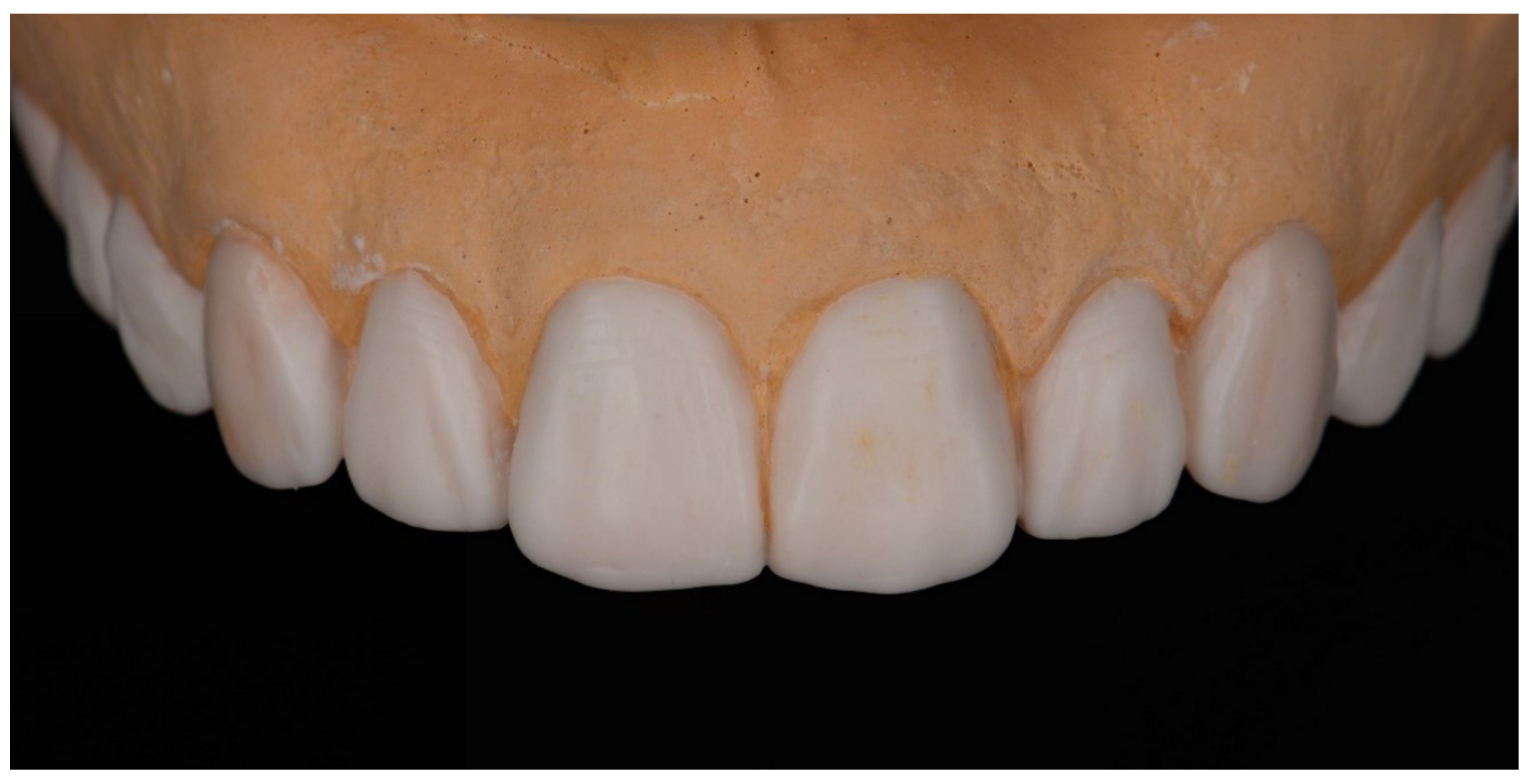

Figure 5. A diagnostic wax up. 


\subsection{Treatment Rendered}

To minimize the discrepancy between anterior and posterior gingival levels, crown lengthening was performed on the maxillary first molars and first and second premolars as well as mandibular incisors (Figure 6). The diagnostic wax up with idealized gingival zenith levels was used to fabricate a clear surgical stent that served as a visual aid for the resident during surgery to approximate final gingival margins. After 16 weeks of healing and tissue maturation, teeth were prepared for full or partial coverage bonded all-ceramic restorations. Due to the circumferential nature of the fluorosis-affected enamel on the maxillary teeth, all of the preparations in the maxillary arch were for full coverage crowns. On the other hand, the lingual surfaces of the mandibular dentition were generally intact. Therefore, the mandibular posterior teeth were prepared for partial coverage restorations sparing the lingual surfaces, while the anterior teeth were prepared for facial veneers (Figure 7a,b). Whenever possible, every attempt was made to maximize the amount of enamel substrate remaining for optimal bonding of the final restorations, and margins were kept supragingival. The extent of interproximal demineralization and caries dictated the interproximal depths of the preparation finish lines. The tooth preparations were completed following standard protocols to achieve adequate reduction for the material thickness required for lithium disilicate (eMax, Ivoclar Vivadent) [32].

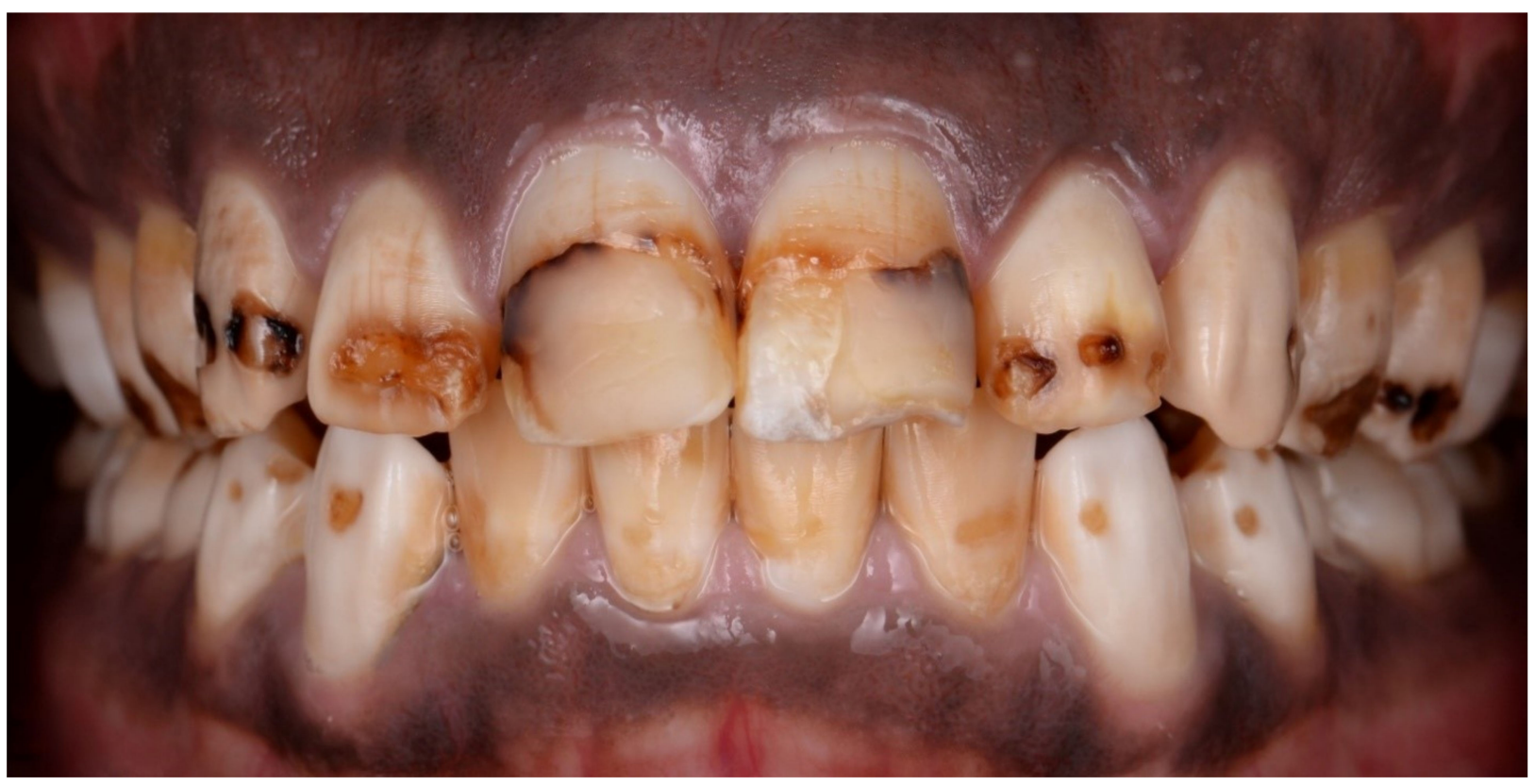

Figure 6. Patient's dentition after maxillary crown lengthening procedures.

Discoloration in several localized areas of the underlying tooth structure on the maxillary anterior teeth was noted. These areas were prepared minimally with a coarse round diamond bur and restored with a light-cured composite build-up material (Photocore, Kuraray) to minimize the potential for the dark substrate showing through the ceramic crown. A PMMA shell (SR Ivocron, Ivoclar Vivadent) was fabricated from the original wax up and relined intraorally using the palate as a reference for maxillary provisionalization, and the unprepared third molars and palatal surfaces of the canines were used as reference for the mandible (Figure 8). 


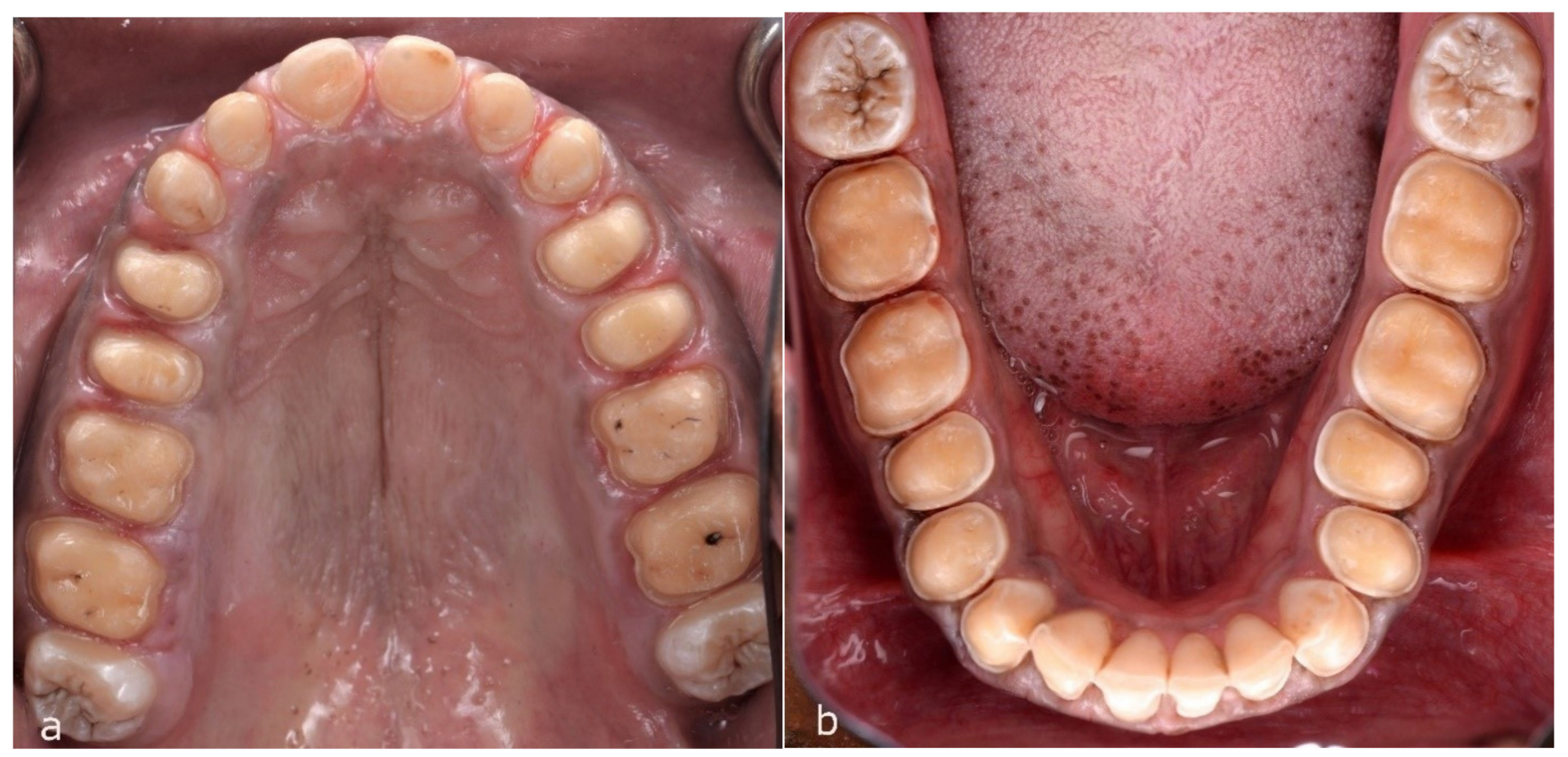

Figure 7. Final preparations: (a) maxillary abutment preparations and (b) mandibular abutment preparations.

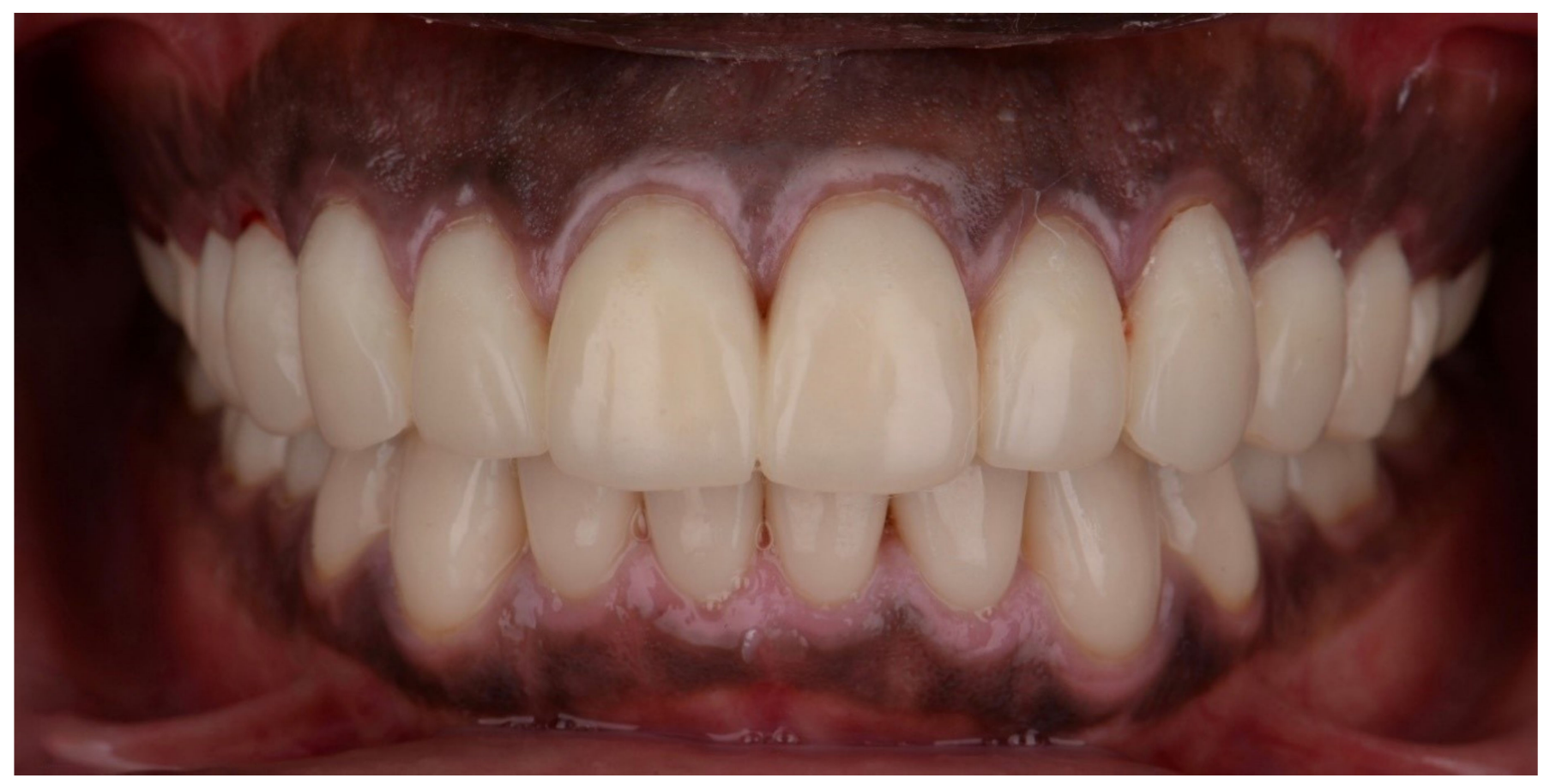

Figure 8. Maxillary and mandibular dentition after provisionalization with PMMA shell.

Final impressions were taken with a polyvinyl siloxane material (Aquasil, Dentsply Sirona), and definitive pressed lithium disilicate restorations (eMax, Ivoclar Vivadent) were fabricated following the contour and occlusion of the provisional restorations (Figure 9a,b). A cutback technique was utilized to fabricate anterior restorations with incisal translucency and areas of characterization. 

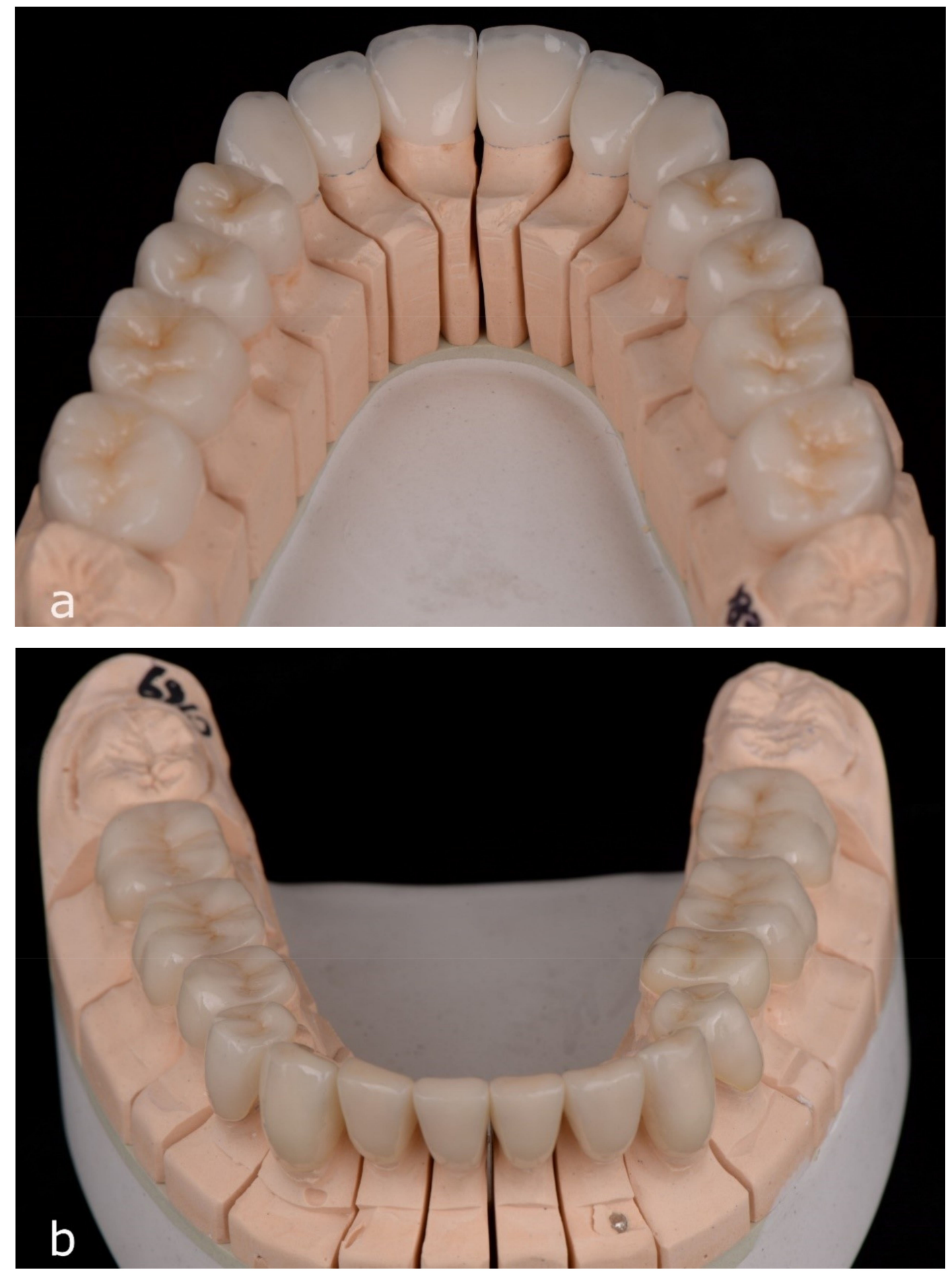

Figure 9. Final restorations with pressed lithium disilicate: (a) maxillary final restorations and (b) mandibular final restorations.

Manufacturer suggestions were followed to adhesively bond the restorations to enamel and dentin. This included a selective-etch technique with an 8th generation bonding agent (Scotchbond Universal Adhesive, $3 \mathrm{M}$ ) and a compatible resin cement system (RelyX Ultimate, $3 \mathrm{M}$ ). The intaglio surfaces of the restorations were treated with $9 \%$ hydrofluoric acid, rinsed, and dried. A silane coupling agent (Silane, Ultradent) was applied to this surface for $60 \mathrm{~s}$ and air-dried. The remaining enamel surfaces of the preparations were treated with $35 \%$ phosphoric acid (Ultra-etch, Ultradent) for $15 \mathrm{~s}$, rinsed, and dried. An 8th generation bonding agent (Scotchbond Universal Adhesive, $3 \mathrm{M}$ ) was applied to the 
preparations for $20 \mathrm{~s}$ and air-thinned. A dual-cure resin cement (RelyX Ultimate, $3 \mathrm{M}$ ) was then applied to the intaglio surface of each restoration. The restorations were fullyseated onto the pretreated preparations and spot cured. The gross excess of resin cement was removed, and the restorations were fully light-cured on all accessible surfaces. Final removal of excess cement was completed with scalers, and minor refinements were made to the occlusion to achieve even bilateral contacts and a mutually protected occlusal scheme.

Upon completion of treatment, a significant improvement in the patient's dental function and esthetics was achieved. The patient was delighted with the esthetic results, including a smile line harmonious with facial structures and a complete masking of the underlying fluorotic structures (Figure 10a-c).
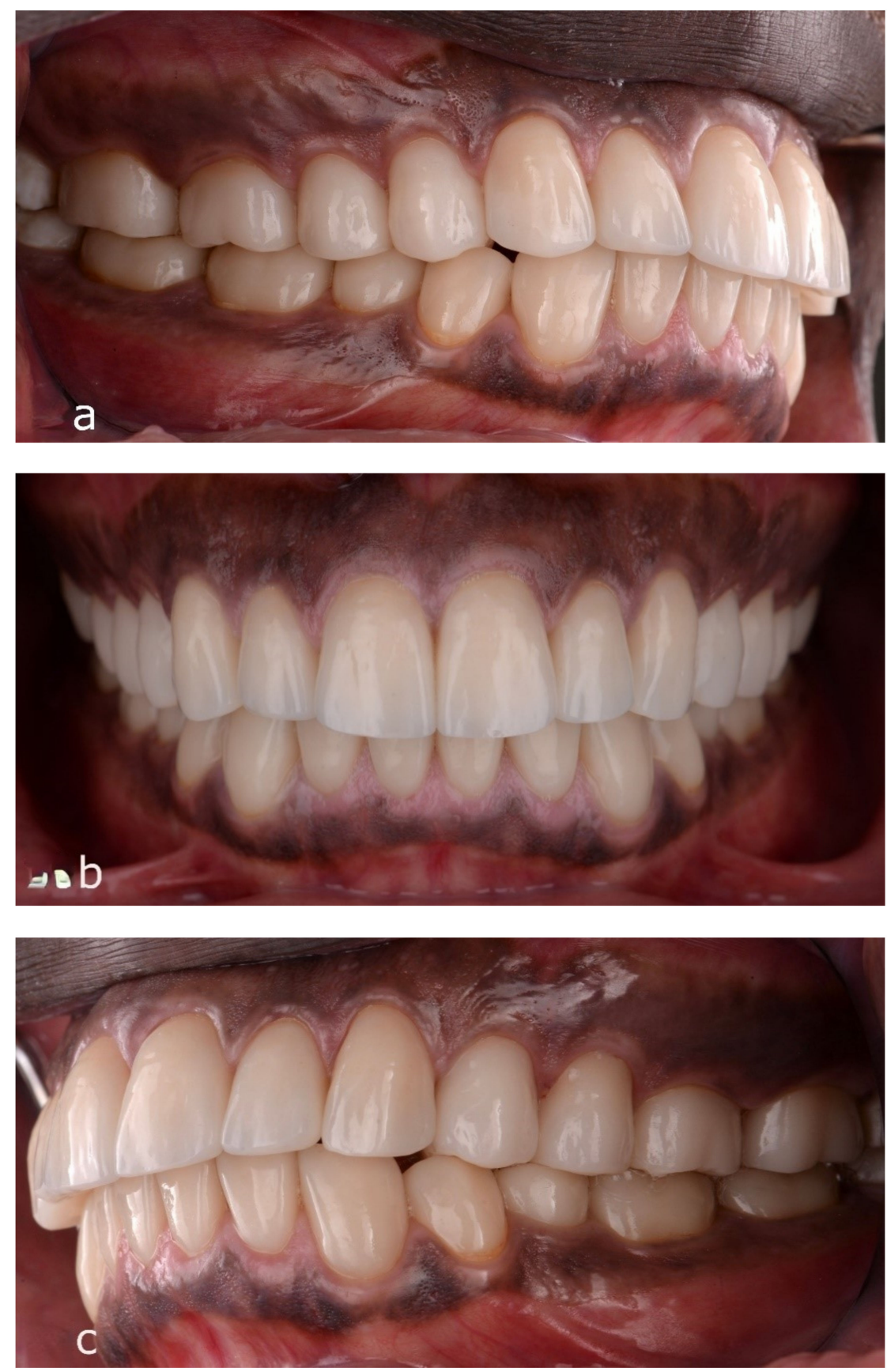

Figure 10. Final restorations bonded using a selective-etch technique: (a) right lateral view, (b) frontal view, and (c) left lateral view. 


\subsection{Follow Up}

Following delivery, a hard acrylic maxillary occlusal guard was fabricated for the patient with centric stops in the posterior dentition, light contact in the anterior dentition, canine disclusion during laterotrusion, and anterior guidance in protrusion. The patient was kept on a six-month recall schedule to promote a healthy dentition and periodontium (Figure 11a,b). At the 2-year follow-up, the esthetic and functional results of the fullmouth rehabilitation were noted to be well maintained, with the patient presenting no complications with the restorations nor the underlying dentition.
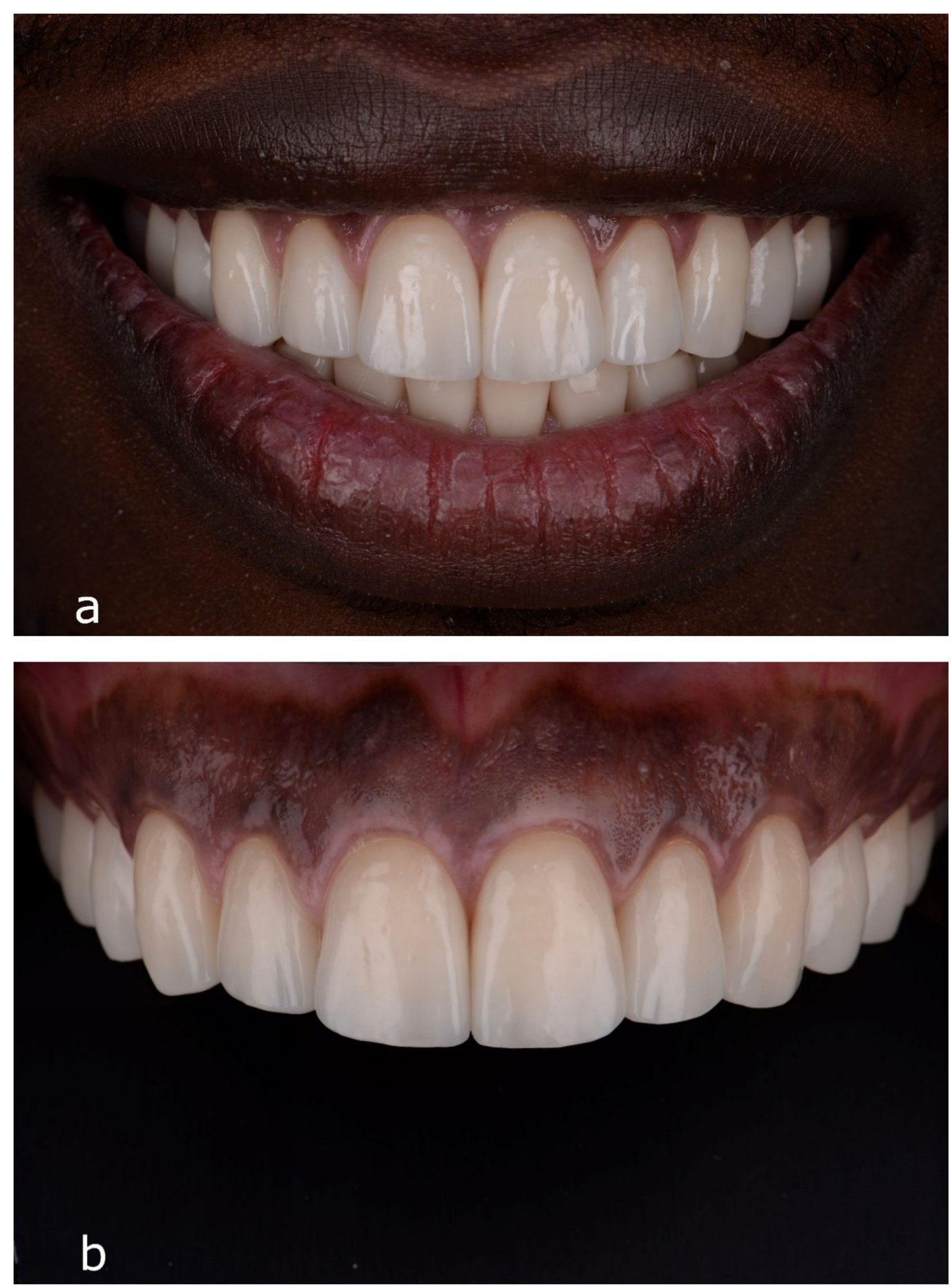

Figure 11. Six-month follow-up: (a) patient at full smile with final restorations and (b) retracted frontal view of the final maxillary anterior restorations. 


\section{Discussion}

Various treatment options have been recommended for the treatment of dental fluorosis ranging from minimally invasive treatments, like tooth whitening or micro-abrasion, to more extensive prosthetic rehabilitations with fixed restorations [25]. The treatment decision depends on the extent and severity of the condition, as well as the patient's esthetic expectations. In mild to moderate cases, the less invasive modalities should be attempted first, especially when considering the fact that the esthetics may improve over time as the patient enters into young adulthood [14]. In severe cases of dental fluorosis, the enamel has a tendency to flake and fracture, which results in significant discoloration and substantial defects in the enamel structure necessitating the use of indirect restorations.

The effects of fluorosis can vary widely by tooth within an individual [3]. In the present case, the patient presented with severe fluorosis affecting a majority of the maxillary dentition and several mandibular posterior teeth, with the remaining teeth exhibiting moderate fluorosis with the exception of the third molars. Due to this mixed presentation, several treatment alternatives were considered. One option was to utilize a combination of both direct and indirect restorations with a majority of the maxillary teeth and mandibular posterior teeth receiving full or partial coverage indirect restorations, and the mandibular anterior teeth being restored with direct restorations, resin infiltration, and bleaching. However, this would have limited the ability to idealize the occlusal plane, manage the crowding, and effectively address the esthetic concerns of the patient. Pre-prosthetic orthodontics were discussed to correct some of these issues and allow for a more conservative approach for restoring the moderately affected dentition with direct restorations. However, the patient declined orthodontic treatment, and bonded all-ceramic restorations were deemed necessary to achieve our treatment objectives. In addition, pre-prosthetic crown lengthening was performed on the maxillary first molars and first and second premolars, as well as the mandibular anterior teeth to correct the significant gingival marginal discrepancies in relation to the adjacent teeth. As the gingival discrepancies stemmed from the altered passive eruption of these teeth, any exposure of the root surfaces could be avoided during this procedure.

Lithium disilicate was selected as the restorative material for this case due to its proven clinical success [33,34], the need for predictable bonding to enamel and dentin [35], favorable esthetics, and more conservative preparation requirements. Recent studies indicate that posterior lithium disilicate partial coverage restorations can be successful at thicknesses of less than $1 \mathrm{~mm}$ when adhesively bonded [32,36,37]. In the present case, considering the fluorosed nature of the tooth substrate, it was deemed necessary to prepare the occlusal surfaces of the posterior teeth to achieve a 1-1.2 $\mathrm{mm}$ thickness of restorative space for adequate material strength. With this amount of tooth structure reduction, there were varying amounts of enamel and dentin remaining on the preparations to serve as the bonding substrate. This conservative approach allowed us to avoid excessive removal of occlusal tooth structure, which could have led to hypersensitivity or pulp exposure.

Despite the compromised bonding surfaces of fluorosed enamel, in vitro studies have shown improved bond strengths to enamel when incorporating phosophoric acid etching in moderately and severely fluorosed teeth [26]. This results in the increased penetration of the resin tags creating a thicker resin-enamel interface. In terms of bonding to fluorosed dentin, the degree of fluorosis has no adverse effects on the shear bond strength to dentin of a two-bottle self-etch system [28]. Thus, in the patient described in this article, a selectiveetch technique was utilized with a universal bonding agent to optimize the bond obtained between the restoration and the underlying tooth structure.

\section{Conclusions}

Dental fluorosis can manifest in a variety of different presentations with varying severity and extent even within a single individual. Thus, the appropriate treatment decision must be reached through a thorough evaluation of the conditions of the affected teeth, as well as the overall functional and esthetic needs of the patient. In this case report, a full- 
mouth rehabilitation was completed with adhesively bonded lithium disilicate restorations to achieve a favorable and predictable treatment outcome. All-ceramic restorations are a viable option in the successful functional and esthetic management of severely fluorosed dentition.

Author Contributions: Conceptualization, J.D.L. and C.L.; treatment, C.L. and S.P.; writing—original draft preparation, N.I. and J.D.L.; writing-review and editing, S.J.L.; supervision, S.J.L. All authors have read and agreed to the published version of the manuscript.

Funding: This case report received no external funding.

Institutional Review Board Statement: This was a case report, and not a study. No IRB approval was required.

Informed Consent Statement: Informed consent was obtained from the patient described.

Acknowledgments: We would like to acknowledge Cusp Dental Laboratory (Malden, MA, USA) for the lab work.

Conflicts of Interest: The authors declare no conflict of interest.

\section{References}

1. Den Besten, P.K. Dental fluorosis: Its use as a biomarker. Adv. Dent. Res. 1994, 8, 105-110. [CrossRef]

2. Den Besten, P.K.; Thariani, H. Biological mechanisms of fluorosis and level and timing of systemic exposure to fluoride with respect to fluorosis. J. Dent. Res. 1992, 71, 1238-1243. [CrossRef]

3. Thylstrup, A.; Fejerskov, O. clinical appearance of dental fluorosis in permanent teeth in relation to histologic changes. Community Dent. Oral. Epidemiol. 1978, 6, 315-328. [CrossRef] [PubMed]

4. Cunha-Cruz, J.; Nadanovsky, P. Dental fluorosis increases caries risk. J. Evid. Based. Dent. Pract. 2005, 5, 170-171. [CrossRef]

5. Ibrahim, Y.E.; Bjorvatn, K.; Birkeland, J.M. Caries and dental fluorosis in a 0.25 and a 2.5 ppm fluoride area in the Sudan. Int. J. Paediatr. Dent. 1997, 7, 161-166. [CrossRef]

6. Ramesh, M.; Narasimhan, M.; Krishnan, R.; Aruna, R.M.; Kuruvilla, S. The effect of fluorosis on human teeth under light microscopy: A cross-sectional study. J. Oral. Maxillofac. Pathol. 2017, 21, 345-350. [CrossRef] [PubMed]

7. Vieira, A.; Hancock, R.; Dumitriu, M.; Schwartz, M.; Limeback, H.; Grynpas, M. How does fluoride affect dentin microhardness and mineralization? J. Dent. Res. 2005, 84, 951-957. [CrossRef]

8. Rozier, R.G. Epidemiologic indices for measuring the clinical manifestations of dental fluorosis: Overview and critique. Adv. Dent. Res. 1994, 8, 39-55. [CrossRef]

9. Chankanka, O.; Levy, S.M.; Warren, J.J.; Chalmers, J.M. A literature review of aesthetic perceptions of dental fluorosis and relationships with psychosocial aspects/oral health-related quality of life. Community Dent. Oral. Epidemiol. 2010, 38, 97-109. [CrossRef]

10. Whelton, H.; Crowley, E.; O'Mullane, D.; Donaldson, M.; Kelleher, V.; Cronin, M. Dental caries and enamel fluorosis among the fluoridated and non-fluoridated populations in the Republic of Ireland in 2002. Community Dent. Health 2004, 21, 37-44.

11. Dean, H.T.; McKay, F.S. Production of Mottled Enamel Halted by a Change in Common Water Supply. Am. J. Public Health Nations Health 1939, 29, 590-596. [CrossRef]

12. Clark, D.C. Trends in prevalence of dental fluorosis in North America. Community Dent. Oral. Epidemiol. 1994, $22,148-152$. [CrossRef]

13. McDonagh, M.S.; Whiting, P.F.; Wilson, P.M.; Sutton, A.J.; Chestnutt, I.; Cooper, J.; Misso, K.; Bradley, M.; Treasure, E.; Kleijnen, J. Systematic review of water fluoridation. BMJ 2000, 321, 855-859. [CrossRef]

14. Curtis, A.M.; Levy, S.M.; Cavanaugh, J.E.; Warren, J.J.; Kolker, J.L.; Weber-Gasparoni, K. Decline in Dental Fluorosis Severity during Adolescence: A Cohort Study. J. Dent. Res. 2020, 99, 388-394. [CrossRef]

15. Srivastava, S.; Flora, S.J.S. Fluoride in Drinking Water and Skeletal Fluorosis: A Review of the Global Impact. Curr. Environ. Health Rep. 2020, 7, 140-146. [CrossRef]

16. Malago, J.; Makoba, E.; Muzaka, A.N.N. Fluoride levels in surface and groundwater in Africa: A review. Am. J. Water Sci. Eng. 2017, 3, 1-17. [CrossRef]

17. Keshavarz, S.; Ebrahimi, A.; Nikaeen, M. Fluoride exposure and its health risk assessment in drinking water and staple food in the population of Dayyer, Iran, in 2013. J. Educ. Health Promot. 2015, 4, 72.

18. Demelash, H.; Beyene, A.; Abebe, Z.; Melese, A. Fluoride concentration in ground water and prevalence of dental fluorosis in Ethiopian Rift Valley: Systematic review and meta-analysis. BMC Public Health 2019, 19, 1298. [CrossRef] [PubMed]

19. Hawley, G.M.; Ellwood, R.P.; Davies, R.M. Dental caries, fluorosis and the cosmetic implications of different TF scores in 14-year-old adolescents. Community Dent. Health 1996, 13, 189-192. [PubMed]

20. Nair, R.; Chuang, J.C.; Lee, P.S.; Leo, S.J.; Yang, N.Q.; Yee, R.; Tong, H.J. Adult perceptions of dental fluorosis and select dental conditions-an Asian perspective. Community Dent. Oral Epidemiol. 2016, 44, 135-144. [CrossRef] 
21. Lalumandier, J.A.; Rozier, R.G. Parents' satisfaction with children's tooth color: Fluorosis as a contributing factor. J. Am. Dent. Assoc. 1998, 129, 1000-1006. [CrossRef]

22. Bouschor, C.F. Bleaching fluorosis stained teeth. N. M. Dent. J. 1965, 16, 33-34.

23. Pontes, D.G.; Correa, K.M.; Cohen-Carneiro, F. Re-establishing esthetics of fluorosis-stained teeth using enamel microabrasion and dental bleaching techniques. Eur. J. Esthet. Dent. 2012, 7, 130-137. [PubMed]

24. Di Giovanni, T.; Eliades, T.; Papageorgiou, S.N. Interventions for dental fluorosis: A systematic review. J. Esthet. Restor. Dent. 2018, 30, 502-508. [CrossRef]

25. Akpata, E.S. Occurrence and management of dental fluorosis. Int. Dent. J. 2001, 51, 325-333. [CrossRef]

26. Weerasinghe, D.S.; Nikaido, T.; Wettasinghe, K.A.; Abayakoon, J.B.; Tagami, J. Micro-shear bond strength and morphological analysis of a self-etching primer adhesive system to fluorosed enamel. J. Dent. 2005, 33, 419-426. [CrossRef]

27. Ermis, R.B.; De Munck, J.; Cardoso, M.V.; Coutinho, E.; Van Landuyt, K.L.; Poitevin, A.; Lambrechts, P.; Van Meerbeek, B. Bonding to ground versus unground enamel in fluorosed teeth. Dent. Mater. 2007, 23, 1250-1255. [CrossRef] [PubMed]

28. Ermis, R.B.; Gokay, N. Effect of fluorosis on dentine shear bond strength of a self-etching bonding system. J. Oral Rehabil. 2003, 30, 1090-1094. [CrossRef]

29. Farid, H.; Khan, F.R. Clinical management of severe fluorosis in an adult. BMJ Case Rep. 2012, 2012, bcr2012007138. [CrossRef] [PubMed]

30. Morimoto, S.; Rebello de Sampaio, F.B.; Braga, M.M.; Sesma, N.; Ozcan, M. Survival Rate of Resin and Ceramic Inlays, Onlays, and Overlays: A Systematic Review and Meta-Analysis. J. Dent. Res. 2016, 95, 985-994. [CrossRef] [PubMed]

31. Sailer, I.; Makarov, N.A.; Thoma, D.S.; Zwahlen, M.; Pjetursson, B.E. All-ceramic or metal-ceramic tooth-supported fixed dental prostheses (FDPs)? A systematic review of the survival and complication rates. Part I: Single crowns (SCs). Dent. Mater. 2015, 31, 603-623. [CrossRef]

32. Luciano, M.; Francesca, Z.; Michela, S.; Tommaso, M.; Massimo, A. Lithium disilicate posterior overlays: Clinical and biomechanical features. Clin. Oral Investig. 2020, 24, 841-848. [CrossRef] [PubMed]

33. Malament, K.A.; Natto, Z.S.; Thompson, V.; Rekow, D.; Eckert, S.; Weber, H.P. Ten-year survival of pressed, acid-etched e.max lithium disilicate monolithic and bilayered complete-coverage restorations: Performance and outcomes as a function of tooth position and age. J. Prosthet. Dentistry 2019, 121, 782-790. [CrossRef] [PubMed]

34. Pieger, S.; Salman, A.; Bidra, A.S. Clinical outcomes of lithium disilicate single crowns and partial fixed dental prostheses: A systematic review. J. Prosthet. Dent. 2014, 112, 22-30. [CrossRef] [PubMed]

35. Rojpaibool, T.; Leevailoj, C. Fracture Resistance of Lithium Disilicate Ceramics Bonded to Enamel or Dentin Using Different Resin Cement Types and Film Thicknesses. J. Prosthodont. 2017, 26, 141-149. [CrossRef]

36. Sasse, M.; Krummel, A.; Klosa, K.; Kern, M. Influence of restoration thickness and dental bonding surface on the fracture resistance of full-coverage occlusal veneers made from lithium disilicate ceramic. Dent. Mater. 2015, 31, 907-915. [CrossRef] [PubMed]

37. Baldissara, P.; Monaco, C.; Onofri, E.; Fonseca, R.G.; Ciocca, L. Fatigue resistance of monolithic lithium disilicate occlusal veneers: A pilot study. Odontology 2019, 107, 482-490. [CrossRef] 